\title{
Code-Aware Quantizer Design for Finite-Precision Min-Sum Decoders
}

\author{
Zeina Mheich*, Thien-Truong Nguyen-Ly*, Valentin Savin* and David Declercq ${ }^{\dagger}$ \\ ${ }^{*}$ CEA-LETI, MINATEC campus, 38054 Grenoble, France, \\ Email: \{Zeina.Mheich,Thientruong.NGUYEN-LY,Valentin.Savin \}@ cea.fr \\ †ETIS-ENSEA, Cergy-Pontoise, France, Email: david.declercq@ensea.fr
}

\begin{abstract}
Classically, the quantization of the soft information supplied to a finite-precision decoder is chosen to optimize a certain criterion which does not depend on the characteristics of the existing code. This work studies code-aware quantizers, for finite-precision min-sum decoders, which optimize the noise threshold of the existing family of Low-Density Parity-Check (LDPC) codes. We propose a code-aware quantizer with lower complexity than that obtained by optimizing all decision levels and approaching its performance, for few quantization bits. We show that code-aware quantizers outperform code-independent quantizers in terms of noise threshold for both regular and irregular LDPC codes. To overcome the error floor behavior of LDPC codes, we propose the design of the quantizer for a target error probability at the decoder output. The results show that the quantizer optimized to get a zero error probability could lead to a very bad performance for practical range of signal to noise ratios. Finally, we propose to design jointly irregular LDPC codes and code-aware quantizers for finite-precision min-sum decoders. We show that they achieve significant decoding gains with respect to LDPC codes designed for infinite-precision belief propagation decoding, but decoded by finite-precision min-sum.
\end{abstract}

\section{INTRODUCTION}

LDPC codes are linear forward error-correction codes, first proposed by Gallager in his $\mathrm{PhD}$ thesis [1]. Irregular LDPC codes proposed later in [2], easily outperform the best turbo codes when the block length of the code is large. This paper focuses on LDPC codes with min-sum decoder, which is more practical to implement than the sum-product decoder at the cost of a slight degradation in performance. The min-sum algorithm uses simple arithmetic (additions) and logical (comparisons) operations; hence it is suitable for hardware implementation. Nowadays, using few bits of precision to represent channel output and the soft information propagating during the iterative decoding process is crucial for high speed applications and for reducing hardware complexity. However, the performance of the decoder will decrease when the number of quantization bits decreases. Therefore, a good design of quantizer at the input of the decoder is necessary to achieve the best performance under a constrained low-precision hardware. Recently, many works are focusing on the field of FPGA implementation of LDPC decoders, see, e.g., [3] and references therein. On the other hand, most of works in literature, consider the design of the quantizer at the channel output independently of the channel code used by the communication system [4], [5].

To evaluate the asymptotic performance of a family of LDPC codes, an iterative process called density evolution (DE) is used, assuming that the Tanner graph of this family is cyclefree. DE can be used to find the maximum level of channel noise, called noise threshold, which can be corrected by a family of LDPC codes using the message passing algorithm. In this work, DE is used as a tool to search for the quantizer that can achieve the best noise threshold for a specific family of LDPC codes. There are few works in literature that analyze the dependency of optimal quantizers on the channel code. In [6], the authors consider the code-dependent quantizers for BI-AWGN channel when regular LDPC codes are used and evaluate the quantizer performance by density evolution. They demonstrate that quantizers that maximize the noise threshold are superior to Lloyd quantizers. Although the authors consider quantized decoder inputs, a belief-propagation decoding was considered with infinite-precision messages exchanged between variable and check nodes in density evolution.

The contributions of this paper are the following. First, we propose a low complexity code-aware quantizer, whose performance approaches that of the code-aware quantizer with decision levels optimized through exhaustive search. We further show that finite-precision min-sum decoders exhibit an asymptotic error-floor phenomenon, which limits the performance of the LDPC code family. Therefore, the code-aware quantizer that maximizes the DE threshold (to get zero error probability at the decoder output), may not be suitable for lower bit error rate. Thus we propose the design of codeaware quantizer for a target bit error rate and we show that the quantizer optimality is highly dependent on the target bit error rate. Finally, we propose to design jointly irregular LDPC codes and code-aware quantizers for finite-precision min-sum.

\section{SYSTEM MODEL}

This paper deals with the point to point communication system as shown in Figure 1. The study could be extended to other channel models. We assume that the coded bits are modulated using a BPSK constellation, $X \in \mathcal{X}=\{+1,-1\}$, and are transmitted over a Gaussian channel with noise variance $\sigma^{2}$. The choice of low-order BPSK modulation is to simplify the analysis of density evolution. At the receiver side, a metric denoted by $L$ is calculated from the channel output $Y$ and then quantized to $\bar{L} \triangleq q(L)$ using a quantizer $q$ to be used as input for the finite-precision min-sum decoder [7] (By abuse of notation, the quantizer is denoted by its associated quantization map $q$ ). Usually, $L$ represents the log-likelihood ratio (LLR). However, in this work, it could be not the LLR. A $n$-bit quantizer $q$ quantifies its input $L$ on $n$ bits. Since the performance of the quantizer will be evaluated according to its resulting noise threshold using density evolution, a symmetricoutput quantizer will be considered for convenience. Thus 
the $n$-bit quantizer has $2^{n}-1$ output values belonging to $\mathcal{Q}_{n}=\{-N, \ldots,-1,0,+1, \ldots,+N\}$, where $N \triangleq 2^{n-1}-1$. The cardinality of the set $\mathcal{Q}_{n}$ is denoted by $\left|\mathcal{Q}_{n}\right|=2 \cdot N+1$. For convenience, the non-negative elements elements of the set $\mathcal{Q}_{n}$ are denoted by $\bar{L}_{i}$ such that $\bar{L}_{i}=i, \forall i \geq 0$. In the following, the reconstruction levels of the $n$-bit quantizer are constrained to the values of $\mathcal{Q}_{n}$ and only the decision levels can be optimized for practical purpose. Since a symmetric quantizer is used, the quantizer can be fully characterized by the non-negative decision levels denoted by $T_{i}, i \in\{1, . ., N\}$, such that $T_{i} \leq T_{i+1}$. By convention $T_{N+1}=+\infty$. The quantizer quantifies its input value $L$ into $q(L)$ such that

$$
\left\{\begin{array}{l}
q(L)=\bar{L}_{i} \quad \text { if } \quad L \in \mathcal{I}_{i} \triangleq\left[T_{i}, T_{i+1}[\right. \\
\left.\left.q(L)=-\bar{L}_{i} \quad \text { if } \quad L \in \mathcal{I}_{-i} \triangleq\right]-T_{i+1},-T_{i}\right], \\
\left.q(L)=\bar{L}_{0} \quad \text { if } \quad L \in \mathcal{I}_{0} \triangleq\right]-T_{1},+T_{1}[
\end{array}\right.
$$

where $\bar{L}_{i}=i \in\{1, \ldots, N\}$. Note that if $T_{i}=T_{i+1}$ the values $\bar{L}_{i}$ and $-\bar{L}_{i}$ are never taken by the quantizer $\forall i \geq 1$. Besides, if $T_{1}=0$, then the value $\bar{L}_{0}$ is never taken by the quantizer.

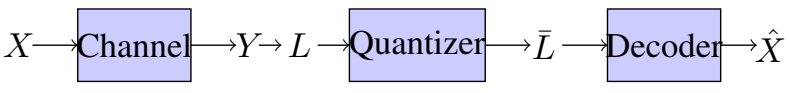

Fig. 1. Point to point communication system with quantized-input decoder

Equation (1) gives the general definition of a quantizer. By a slight abuse of language, we shall use the same terminology to refer to both the optimization criterion and the resulting quantizer.

\section{CODE-AWARE QUANTIZERS}

\section{A. Decision levels quantizer (DL)}

This section investigates code-aware quantizer design for BI-AWGN channel when LDPC codes are used along with min-sum decoding. Our study differs from [6] by the use of finite-precision $L D P C$ decoders where the messages exchanged using density evolution are also quantized on a fixed number of bits as well as the decoder input. Moreover, our study includes both regular and irregular LDPC codes. We refer the reader to [7] for the density evolution equations for finite-precision minsum decoding. The density evolution equations allow to obtain the error probability $P_{e}^{(\ell)}$ at each iteration $\ell$ of the iterative message-passing algorithm. The criterion used to optimize the quantizer is the noise threshold $\sigma_{t h}^{2}$ defined by

$$
\sigma_{t h}^{2}=\sup \left\{\sigma^{2}: \lim _{\ell \rightarrow \infty} P_{e}^{(\ell)}=0\right\}
$$

The finite-precision min-sum decoder is known to have high error floors. Thus a quantizer $q_{1}$ which is better than another quantizer $q_{2}$ for a target bit error rate may be worse than $q_{2}$ for a different target bit error rate. Moreover, in practice the target bit error rate is usually fixed to a practical value (eg. $10^{-5}$ ) because a very low target error probability requires higher SNR in general. Hence, we investigate the optimal quantization which maximizes the noise threshold for zero error probability using density evolution as well as the optimal quantization which maximizes the noise threshold for a target bit error rate. The $\eta$-threshold [8] for a target bit error rate $\eta$ is defined by

$$
\sigma_{t h}^{2}(\eta)=\sup \left\{\sigma^{2}: \lim _{\ell \rightarrow \infty} P_{e}^{(\ell)} \leq \eta\right\}
$$

When $\eta=0$, the $\eta$-threshold will be called "DE threshold" and is equivalent to the threshold defined in (2). The codeaware quantizer design for a target bit error rate $\eta$ requires to solve the following optimization problem

$$
\max _{T_{i}, i=1, \ldots, N} \sigma_{t h}^{2}\left(T_{1}, . ., T_{N} ; \eta\right)
$$

This problem is solved using an exhaustive search method.

\section{B. Gain factor quantizer $(G F)$}

Since the complexity of exhaustive search methods increases with the number of quantization bits, we provide a code-aware quantizer design with lower complexity based on a uni-parametric optimization. The idea is to relax the constraint on the value of $g \triangleq \frac{2}{\sigma^{2}}$ in the expression of the LLR given by $L=g \cdot Y$ and optimize $g$. This case uses the symmetric quantizer such that the non-negative decision levels are given by $T_{i}=\frac{\bar{L}_{i-1}+\bar{L}_{i}}{2}$ for $i=1, \ldots, N$, however the channel output $Y$ is scaled by the gain factor $g$. In other terms, $L$ is quantized to the nearest integer in $\mathcal{Q}_{n}$. To obtain the optimal gain factor for a target bit error rate $\eta$ we should solve the following optimization problem

$$
\max _{g} \sigma_{t h}^{2}(g ; \eta)
$$

Problem (5) is a uni-dimensional optimization problem and is solved using an exhaustive search method.

Remark 1: For GF quantizer, we optimize $g$ such that $L=$ $g \cdot Y$ but the decision levels are fixed to be $T_{i}=\frac{\bar{L}_{i-1}+\bar{L}_{i}}{2}$ for $i=1, \ldots, N$. The GF quantizer gives the same performance of the quantizer with $L=Y$ and $T_{i}=\frac{1}{g} \cdot \frac{\bar{L}_{i-1}+\bar{L}_{i}}{2}$ or the quantizer with $L=\frac{2}{\sigma^{2}} \cdot Y$ and $T_{i}=\frac{2}{g \sigma^{2}} \cdot \frac{\bar{L}_{i-1}+\bar{L}_{i}}{2}$ for $i=1, \ldots, N$.

\section{PERFormance EVAluation}

Throughout this section, we assume that the a posteriori information of the finite-precision min-sum decoder is quantized on $\tilde{n}=n+1$ bits, where $n$ is the number of quantization bits for the a priori information and exchanged messages [7]. The performance of the code-aware quantizers will be compared to that of two code-independent quantizers. The first, denoted by $\mathrm{MI}_{X \bar{L}}$, is the quantizer which maximizes the mutual information between the channel input $X$ and the quantizer output $\bar{L}$ [4]. The second, denoted by $\mathrm{MI}_{L \bar{L}}$, is the quantizer which maximizes the mutual information between the quantizer input $L$ and its output $\bar{L}$ [5].

\section{A. (Semi-) Regular LDPC codes}

TABLE I. DE THRESHOLD OF SOME QUANTIZERS FOR THE FAMILY OF (SEMI-)REGULAR LDPC CODES OF RATE $R$ AND VARIABLE NODE DEGREE $d_{v}=3$, WHEN $n=3$ BITS.

\begin{tabular}{|l|l|l|l|l||l|}
\hline$R$ & $\mathrm{GF}$ & $\mathrm{DL}$ & $\mathrm{MI}_{X \bar{L}}$ & $\mathrm{MI}_{L \bar{L}}$ & $\begin{array}{l}\text { Opt. } g \\
\text { for GF }\end{array}$ \\
\hline $1 / 3$ & 1.0234 & 1.0234 & 1.0116 & 0.9898 & 2.2592 \\
\hline $1 / 2$ & 0.6625 & 0.6625 & 0.6591 & 0.6266 & 2.7726 \\
\hline $2 / 3$ & 0.4399 & 0.4399 & 0.4389 & 0.3934 & 3.1871 \\
\hline $3 / 4$ & 0.3554 & 0.3554 & 0.3544 & 0.2885 & 3.4510 \\
\hline $5 / 6$ & 0.2799 & 0.2799 & 0.2785 & - & 3.8480 \\
\hline
\end{tabular}


Table I shows the noise thresholds $\left(\sigma_{t h}^{2}\right)$ for some LDPC codes of rate $R$ and variable node degree $d_{v}$ when the a priori information and the exchanged messages of the finite-precision min-sum decoder are quantized on $n=3$ bits. Since we have fixed $R$ and $d_{v}$, the check nodes can be all of the same degree (regular code) or not (semi-regular code). The thresholds are given in Table I for $\eta=0$ (zero error rate on the decoder output). The precision on the threshold value is fixed to $10^{-4}$. The results show that the GF quantizer gives the same performance of the DL quantizer of high computational complexity. The quantizer maximizing the mutual information between the channel input and the quantizer output $\left(\mathrm{MI}_{X \bar{L}}\right)$ gives good performance which coincides with the performance of DL for some codes. The quantizer $\mathrm{MI}_{X \bar{L}}$ gives better performance than the quantizer maximizing the mutual information between the input of the quantizer and its output $\mathrm{MI}_{L \bar{L}}$ for regular codes. It can be observed that the quantizer $\mathrm{MI}_{L \bar{L}}$ does not achieve a target zero error probability when the code rate is higher than a certain value (eg. when $R=\frac{5}{6}$ in Table I).

\section{B. Irregular $L D P C$ codes}

In this section, we study the performance of irregular LDPC codes in terms of noise threshold using code-aware and code-independent quantizers. Consider the family of irregular LDPC codes of rate one-half, variable node degree distribution $\lambda(x)=0.23882 x+0.29515 x^{2}+0.03261 x^{3}+0.43342 x^{10}$ and check node degree distribution $\rho(x)=0.43011 x^{6}+0.56989 x^{7}$. This code was shown to be powerful for belief-propagation decoding and has a noise DE threshold of $\sigma_{t h}^{2}=0.9162$ [2]. However, for the finite-precision min-sum decoder, the performance of this code seems to be worse than the regular code of rate one half in the previous section, especially when the number of quantization bits is small as shown in Table II, due to the error floor. The DL quantizer is not considered when $n>3$ due to the complexity of the exhaustive search in this case. Tables III and IV show the $\eta$-threshold for the same irregular code when the number of quantization bits is $n=2$ and 3 respectively and $\eta \in\left\{10^{-3}, 10^{-4}, 10^{-5}, 10^{-10}\right\}$. We observe also that the quantizer $\mathrm{MI}_{L \bar{L}}$ is better than $\mathrm{MI}_{X \bar{L}}$ in most cases contrarily to the case of regular codes in the previous section. In general, we can see that any of the two quantizers $\left(\mathrm{MI}_{X \bar{L}}\right.$ and $\mathrm{MI}_{L \bar{L}}$ ) can be better than the other depending on the values of $\eta$ and $n$ because these quantizers are not code-aware. The code aware quantizer GF can achieve noticeable gains comparing to the best quantizer among the independent-code quantizers depending on $\eta$ and $n$. For $n=2$, GF quantizer has the same performance as the DL quantizer. This may be obvious because there is one variable to optimize in both cases.

$\square$ DL quantizer $\circ$ GF quantizer

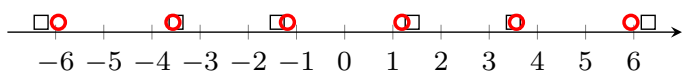

Fig. 2. Decision levels of DL and GF quantizers obtained when $\eta=10^{-5}$, $n=3$ and using the irregular LDPC code of rate one-half in section IV-B

For $n=3$, the performance of GF quantizer is very close to that of DL quantizer. The decision levels obtained for both code-aware quantizers are very close as shown in Fig. 2 when $\eta=10^{-5}$. It is worth to note that in Fig. 2, the decisions levels are given when $L=\frac{2}{\sigma_{t h}^{2}(D L)} \cdot Y$, where $\sigma_{t h}^{2}(D L)$ is the $\eta$-threshold for DL quantizer with $\eta=10^{-5}$, for both quantizers in order to compare them for the same $L$. Then, the decision levels for GF quantizer are given by $T_{i}=\frac{2}{g \cdot \sigma_{t h}^{2}(D L)}$. $\frac{\bar{L}_{i-1}+\bar{L}_{i}}{2}$ for $i=1, \ldots, N$ (cf. Remark 1) where $g$ is the optimal gain factor for $\eta=10^{-5}$ which is given in Table IV.

TABLE II. DE THRESHOLD OF SOME QUANTIZERS FOR THE FAMILY OF IRREGULAR LDPC CODES OF RATE $\frac{1}{2}, \lambda(x)=0.23882 x+0.29515 x^{2}+$ $0.03261 x^{3}+0.43342 x^{10}$ AND $\rho(x)=0.43011 x^{6}+0.56989 x^{7}$.

\begin{tabular}{|l|l|l|l|l||l|}
\hline$n$ & $\mathrm{GF}$ & $\mathrm{DL}$ & $\mathrm{MI}_{X \bar{L}}$ & $\mathrm{MI}_{L \bar{L}}$ & $\begin{array}{l}\text { Opt. } g \\
\text { for GF }\end{array}$ \\
\hline 2 & 0.0560 & 0.0570 & 0.0156 & 0.0525 & 0.5092 \\
\hline 3 & 0.1179 & 0.1179 & 0.0359 & 0.0557 & 0.5135 \\
\hline 4 & 0.2932 & Not given & 0.0759 & 0.0570 & 0.5322 \\
\hline
\end{tabular}

TABLE III. $\quad \eta$-THRESHOLD OF SOME QUANTIZERS FOR THE FAMILY OF IRREGULAR LDPC CODES OF RATE $\frac{1}{2}, \lambda(x)=0.23882 x+0.29515 x^{2}$ $+0.03261 x^{3}+0.43342 x^{10}$ AND $\rho(x)=0.43011 x^{6}+0.56989 x^{7} \cdot n=2$.

\begin{tabular}{|l|l|l|l|l||l|}
\hline$\eta$ & $\mathrm{GF}$ & $\mathrm{DL}$ & $\mathrm{MI}_{X \bar{L}}$ & $\mathrm{MI}_{L \bar{L}}$ & $\begin{array}{l}\text { Opt. } g \\
\text { for GF }\end{array}$ \\
\hline $10^{-3}$ & 0.5362 & 0.5362 & 0.4330 & 0.5107 & 0.7952 \\
\hline $10^{-4}$ & 0.4536 & 0.4536 & 0.2825 & 0.4351 & 0.6146 \\
\hline $10^{-5}$ & 0.3760 & 0.3760 & 0.1947 & 0.3490 & 0.5592 \\
\hline $10^{-10}$ & 0.1868 & 0.1868 & 0.0667 & 0.1704 & 0.5179 \\
\hline
\end{tabular}

TABLE IV. $\eta$-THRESHOLD OF SOME QUANTIZERS FOR THE FAMILY OF IRREGULAR LDPC CODES OF RATE $\frac{1}{2}, \lambda(x)=0.23882 x+0.29515 x^{2}+$ $0.03261 x^{3}+0.43342 x^{10}$ AND $\rho(x)=0.43011 x^{6}+0.56989 x^{7} \cdot n=3$.

\begin{tabular}{|l|l|l|l|l||l|}
\hline$\eta$ & $\mathrm{GF}$ & $\mathrm{DL}$ & $\mathrm{MI}_{X \bar{L}}$ & $\mathrm{MI}_{L \bar{L}}$ & $\begin{array}{l}\text { Opt. } g \\
\text { for } \mathrm{GF}\end{array}$ \\
\hline $10^{-3}$ & 0.6733 & 0.6798 & 0.6668 & 0.6620 & 2.3247 \\
\hline $10^{-4}$ & 0.6600 & 0.6650 & 0.4792 & 0.6590 & 1.5193 \\
\hline $10^{-5}$ & 0.6350 & 0.6384 & 0.3373 & 0.5432 & 1.3183 \\
\hline $10^{-10}$ & 0.4869 & 0.4870 & 0.1182 & 0.2716 & 0.6536 \\
\hline
\end{tabular}

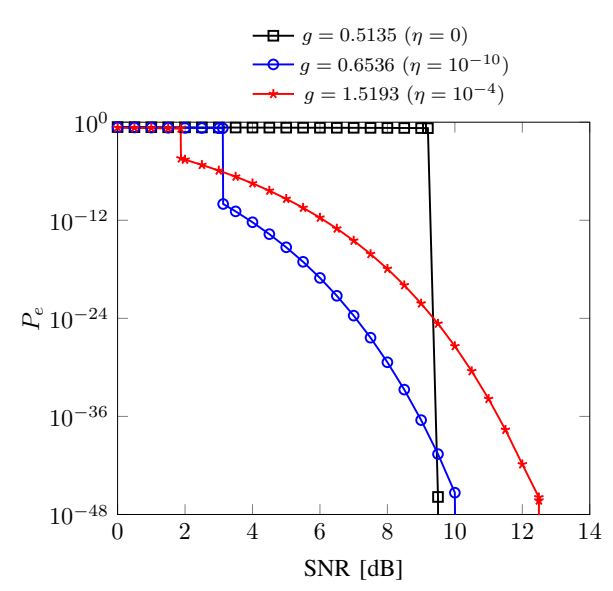

Fig. 3. Error probability $P_{e}$ obtained via DE using the GF quantizer with parameter $g$ as a function of the channel SNR when $n=3$ bits. The value of $\eta$ for which the quantizer is optimal is given between parentheses.

Figure 3 shows the asymptotic error probability (i.e. $P_{e}=$ $\left.\lim _{\ell \rightarrow \infty} P_{e}^{(\ell)}\right)$ at the decoder output obtained via density evolution as a function of the channel SNR for the GF quantizer and 
for different values of the gain factor $g$. It can be observed that the optimal value of $g$ which maximizes the noise threshold (equivalently minimizes the SNR threshold) for a zero target bit error rate has a bad performance for the SNR values smaller than $9 \mathrm{~dB}$. This is due to the error floor in the curve of error probability when the slope changes from a certain value of SNR. Thus, it is necessary to optimize the quantizer for a target error probability to avoid having an error floor behavior in the curve or bad performance at this target value. To the best of our knowledge, this is the first time it is shown that for finiteprecision decoders the error floor is not solely due to specific topologies in the finite-length graph, but also to the "noise" (impreciseness) introduced by the finite precision operations, which manifests itself even for infinite length codes.

\section{Finite length performance of $G F$ quantizer}

In this section, we study the performance of GF quantizer for finite length irregular LDPC codes, with node-degree distribution polynomials $\lambda$ and $\rho$ from Section IV-B. Figure 4 shows the bit error rate (BER) curves, with $n=4$, for infinite length code based on density evolution and for finite length codes with a codeword length $V$, when $\eta=10^{-4}$. Two methods for constructing finite length codes are under consideration. The first method is when the code is constructed randomly (RAND). The second is when the code is constructed using the "progressive edge growth" (PEG) algorithm [9] in order to avoid undesirables graph topologies (such as short cycles, small trapping sets,...). For the infinite length code, one can see that the BER curve is subject to a fall at the $\eta$-threshold SNR for $\eta=10^{-4}$ in figure 4 . Then the curve experiences an error floor starting from a value of BER equal (in general less or equal) to the target value $\eta$ for which the GF quantizer is optimized. Consequently, one can conclude that optimizing the quantizer is crucial to avoid error floor at the target BER. For the finite length code, the curves exhibit a similar behavior to the infinite length performance; however, the curve for the finite length code presents a smooth fall rather than an infinite-slope fall as in the asymptotic case. We observe that the error probability of the finite length code, which is constructed randomly, is lower bounded by the error probability obtained via density evolution during the error floor part and the curves are not exactly matching. This is due to the topology of the finite length code, which makes its performance affected by other factors (eg. trapping sets [10], absorbing sets $[11], \cdots)$. Moreover, we have observed that the minimum distance of the randomly constructed code is very small, such that in the error floor region the decoder converges quite often to a wrong codeword (different from the one actually sent over the channel). Both codes constructed by the PEG algorithm $(V=4000$ and $V=20000)$ have girth equal to 8 and good minimum distance properties. For these codes, in all the simulations that we ran, the decoder never converged to a wrong codeword. Surprisingly, the error probability of the PEG constructed codes in the error floor region is smaller than the one predicted by density evolution. To explain this, we have investigated the edge-perspective joint degree distribution of the constructed codes. If $(\lambda, \rho)$ is the degree distribution pair of the irregular LDPC code ensemble, an implicit assumption made in the classical density evolution is that the fraction of edges connected to a variable-node of degree $i$ and checknodes of degree $j$ is given by $f_{i j}=\lambda_{i} \cdot \rho_{j}$. However, for

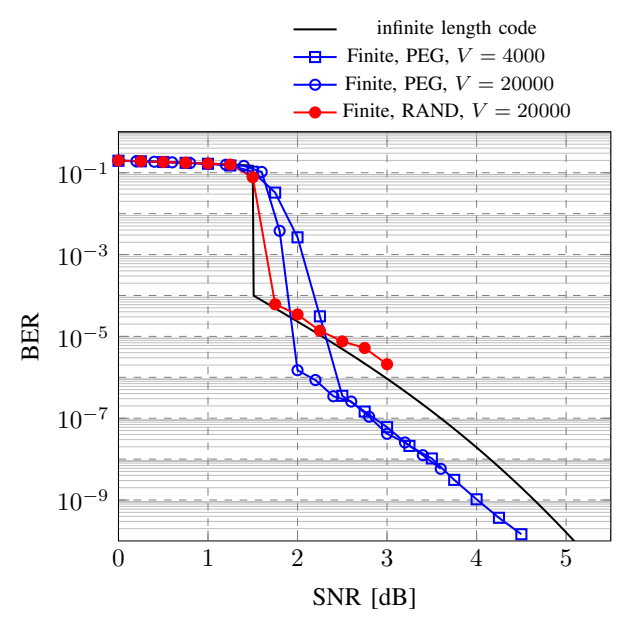

Fig. 4. BER curves for the GF quantizer with finite and infinite length codes when $n=4, \eta=10^{-4}(g=3.8010)$ and using the irregular LDPC code of rate one-half in section IV-B

the PEG constructed codes, we observed a mismatch between the constructed $f_{i j}$ distribution and the theoretical one (even if the constructed $\lambda$ and $\rho$ distributions are very close to the theoretical ones). To overcome this issue, future works should consider the density evolution for multi-edge type LDPC [12].

\section{Irregular LDPC code design for finite-precision decoders}

It was shown in section IV-B that irregular codes designed for infinite-precision decoders based on belief propagation decoding may lead to very bad thresholds when used for finiteprecision min-sum decoders (see Table II). In this section, we propose to design irregular LDPC codes for finite-precision min-sum decoders operating with a certain (small) number of quantization bits $n$ (for the a priori information and exchanged messages) and $\tilde{n}$ (for the a posteriori information). The design of a code for a fixed rate, consists in finding the optimal degree distribution pairs, usually for some fixed maximum variable node and check node degrees $\left(d_{v}\right.$ and $\left.d_{c}\right)$ to simplify the optimization. In our simulations, we have chosen to fix the maximum variable node degree $d_{v}=11$. Ideally, we should consider a variable-node degree distribution of the form $\lambda(x)=\sum_{i=2}^{11} \lambda_{i} x^{i-1}$ and optimize all $\lambda_{i}$. However, this will make the optimization very complex due to the large number of variables. Thus, we have considered a variable-node degree distribution of the form $\lambda(x)=\lambda_{2} x+\lambda_{3} x^{2}+\lambda_{4} x^{3}+\lambda_{11} x^{10}$ which has the same form of the irregular code in section IV-B. The code rate $R$ is fixed to one half. The check-node degree distribution $\rho$ is chosen to be semi-regular, according to the value of $\lambda$ and the coding rate $R$. We consider a GF quantizer, with a gain factor $g$ optimized jointly with the degree distribution pair to maximize the $\eta$-threshold for a target error probability equal to $\eta$. The optimization problem under consideration, for fixed $n$ and $R$, is the following:

$$
\begin{aligned}
\max _{\lambda(x), g} & \sigma_{t h}^{2}(\lambda(x), \rho(x), g ; \eta) \\
\text { subject to: } & \sum_{i} \lambda_{i}=1,0 \leq \lambda_{i} \leq 1 \quad \forall i, g>0
\end{aligned}
$$


where $\rho$ is the unique (semi-)regular polynomial such that

$$
\int_{0}^{1} \rho(x) d x=(1-R) \cdot \int_{0}^{1} \lambda(x) d x
$$

Optimization techniques to solve such problems were described in [2]. In our simulations, we have used "differential evolution" method to solve this problem. Table V gives some good codes found by solving problem (6) for $n=2,3,4$ and $\eta=10^{-10}$. Figure 5 shows the asymptotic error probability as function of the channel SNR for the three codes optimized for the finite-precision min-sum decoding with $n \in\{2,3,4\}$ (Table V) and the code optimized for belief propagation decoding from Section IV-B, but decoded by finite precision min-sum with $n \in\{2,3,4\}$. We observe that noticeable gains of $4.63 \mathrm{~dB}, 1.36 \mathrm{~dB}$, and $0.46 \mathrm{~dB}$ in SNR thresholds are obtained with respect to optimized code for belief propagation decoding, when $n=2,3$ and 4 respectively. Thus, it is crucial to consider the "finite-precision" property of the decoder to design irregular LDPC codes for finite-precision min-sum decoders. We observe in table $\mathrm{V}$ that when $n=2$, the optimized code is very similar to a semi-regular code with $R=\frac{1}{2}$ and $d_{v}=3$, since $\lambda_{3} \approx 1$. The $\eta$-threshold obtained for $n=2$ $\left(\sigma_{t h}^{2}=0.5423889\right)$ is also close to that of the (semi-)regular code of rate one-half with $d_{v}=3$. In table $\mathrm{V}$, we observe that the optimized codes exhibit higher irregularity with increasing $n$. Thus, we conclude that (semi-)regular LDPC codes achieve good thresholds for finite-precision decoders with a very few number of quantization bits.

TABLE V. GOOD DEGREE DISTRIBUTION PAIRS OF RATE 1/2 WHEN $n \in\{2,3,4\}$, AND $\eta=10^{-10}$.

\begin{tabular}{|l|l|l|l|}
\hline$n$ & 2 & 3 & 4 \\
\hline \hline$\lambda_{2}$ & 0 & 0.000276893 & 0.24805492 \\
\hline$\lambda_{3}$ & 0.95587734 & 0.876706068 & 0.49037245 \\
\hline$\lambda_{4}$ & 0.00015006 & 0.000000024 & 0.00000559 \\
\hline$\lambda_{11}$ & 0.04397260 & 0.123017015 & 0.26156704 \\
\hline \hline$\rho_{6}$ & 0.775877 & 0.37470064 & 0.53656845 \\
\hline$\rho_{7}$ & 0.224123 & 0.62529936 & 0.46343155 \\
\hline \hline$g$ & 1.51 & 2.776 & 1.4 \\
\hline \hline$\sigma_{t h}^{2}$ & 0.5423889 & 0.6660461 & 0.717987 \\
\hline \hline SNR $_{t h}[\mathrm{~dB}]$ & 2.656892 & 1.764957 & 1.438834 \\
\hline
\end{tabular}

\section{CONCLUSION}

In this paper we investigated the design of code-aware quantizers for finite-precision min-sum decoders which maximize the noise threshold of the existing family of LDPC code. We proposed a low complexity code-aware quantizer based on scaling a linear quantizer. Besides the quantizer itself, one of the main contributions of the paper was to show that we can use a low complexity quantizer, instead of the quantizer whose all decision levels are optimized exhaustively, with a very small loss in performance. Moreover, we proposed to design the code-aware quantizer for a target BER and it was shown that this can prevent error floor phenomenon at the target BER. Finally, we proposed the joint design of good irregular LDPC code and code-aware quantizer for a min-sum decoder with finite precision resulting into important gain in threshold with respect to irregular LDPC codes designed for infinite-precision belief propagation, but decoded by finite-precision min-sum.

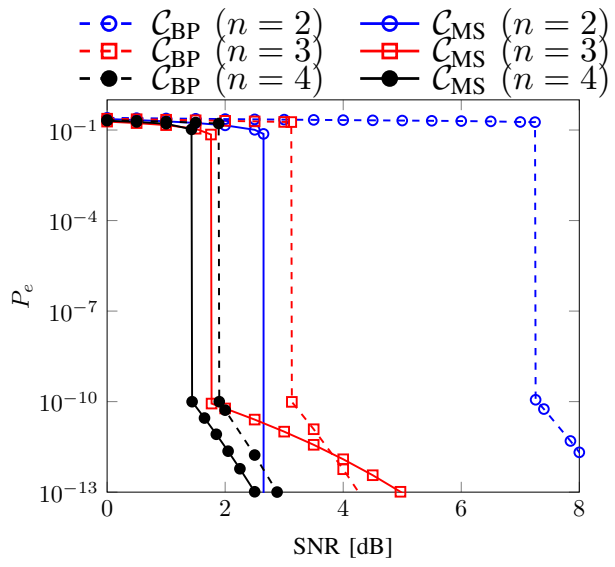

Fig. 5. Error probability $P_{e}$ obtained via density evolution using the GF quantizer as a function of the channel SNR for $\eta=10^{-10}$ and $n \in\{2,3,4\}$. For each $n$, the error probability is plotted for $\mathcal{C}_{\mathrm{MS}}$, the optimized code for the fixed-point min sum decoder given in table $\mathrm{V}$ and $\mathcal{C}_{\mathrm{BP}}$, the optimized code for belief propagation decoding given in section IV-B

This paper can be considered as a benchmark for practical implementation of LDPC codes on a finite precision hardware.

\section{ACKNOWLEDGMENTS}

The authors acknowledge support from the European H2020 Work Programme, project mmMagic, and the FrancoRomanian (ANR-UEFISCDI) Joint Research Programme "Blanc-2013", project DIAMOND.

\section{REFERENCES}

[1] R. G. Gallager, Low-Density Parity-Check Codes. Cambridge, MA:MIT Press, 1963.

[2] T. J. Richardson, M. A. Shokrollahi, and R. L. Urbanke, "Design of capacity-approaching irregular low-density parity-check codes," IEEE Trans. on Information Theory, vol. 47, no. 2, pp. 619-637, Feb 2001.

[3] A. Balatsoukas-Stimming and A. Dollas, "FPGA-based design and implementation of a multi-GBPS LDPC decoder," in proc. of FPL, Aug 2012, pp. 262-269.

[4] A. Liveris and C. Georghiades, "On quantization of low-density paritycheck coded channel measurements," in proc. of IEEE Global Telecommunications Conference, vol. 3, Dec 2003, pp. 1649-1653 vol.3.

[5] C. Novak, P. Fertl, and G. Matz, "Quantization for soft-output demodulators in bit-interleaved coded modulation systems," in proc. of IEEE ISIT, June 2009, pp. 1070-1074.

[6] J. Cho, J. Kim, and W. Sung, "Optimal Output Quantization of Binary Input AWGN Channel for Belief-Propagation Decoding of LDPC Codes," in proc. of IEEE SiPS, Oct 2012, pp. 282-287.

[7] C. K. Ngassa, V. Savin, and D. Declercq, "Min-sum-based decoders running on noisy hardware," in proc. of IEEE Global Communications Conference, Dec 2013, pp. 1879-1884.

[8] L. R. Varshney, "Performance of LDPC codes under faulty iterative decoding," IEEE Trans. Inf. Theory, vol. 57, no. 7, pp. 4427-4444, July 2011.

[9] X.-Y. Hu, E. Eleftheriou, and D. Arnold, "Regular and irregular progressive edge-growth tanner graphs," IEEE Transactions on Information Theory, vol. 51, no. 1, pp. 386-398, Jan 2005.

[10] T. Richardson, "Error floors of LDPC codes," in Proc. 41st Allerton Conf. on Comm., Control, and Computing, USA, Oct. 2003.

[11] Z. Zhang, L. Dolecek, B. Nikolic, V. Anantharam, and M. Wainwright, "Investigation of error floors of structured low-density parity-check codes by hardware emulation," in IEEE GLOBECOM, Nov 2006.

[12] T. Richardson and R. Urbanke, "Multi-Edge Type LDPC Codes," Tech. Rep., 2004, submitted IEEE IT. 